Research Paper

\title{
Evaluation of Breast Cancer Stem Cells and Intratumor Stemness Heterogeneity in Triple-negative Breast Cancer as Prognostic Factors
}

\author{
Fang Yang, Lulu Cao, Zijia Sun, Juan Jin, Hehui Fang, Wenwen Zhang, Xiaoxiang Guan ${ }^{\bowtie}$ \\ Department of Medical Oncology, Jinling Hospital, Medical School of Nanjing University, Nanjing, 210002, P.R. China \\ $\square$ Corresponding author: Professor Xiaoxiang Guan. Addresses: Department of Medical Oncology, Jinling Hospital, Medical School of Nanjing University, 305 \\ East Zhongshan Road, Nanjing, Jiangsu Province, 210002, P. R. China. E-mail: xguan@nju.edu.cn.
}

() Ivyspring International Publisher. Reproduction is permitted for personal, noncommercial use, provided that the article is in whole, unmodified, and properly cited. See http://ivyspring.com/terms for terms and conditions.

Received: 2016.07.17; Accepted: 2016.10.10; Published: 2016.12.07

\begin{abstract}
Triple-negative breast cancer (TNBC) is a tumor subtype with aggressive behavior and poor clinical outcome for lacking effective therapies. Breast cancer stem cells (BCSCs) have been suggested to have tumor-initiating properties, but it remains unclear whether their presence contributes to the increased aggressiveness and poor prognosis of TNBC. Also, the breast cancers display frequent inter- and intra-tumor heterogeneity, which adds the complexity in diagnosis and predicting prognosis. Here we investigated the clinical relevance and prognostic value of the BCSC markers, CD44+/CD24-, aldehyde dehydrogenase family 1 member Al (ALDHIAl) and CDI33 in 88 TNBC cases. We found that a few patients displayed spatial heterogeneity of the BCSC markers in expression, which was defined as intratumor stemness heterogeneity (ITSH) below. There was no significant correlation between any BCSC marker alone or ITSH and progression-free survival (PFS). Interestingly, the combined BCSC phenotype by CD44+/CD24- and ALDH1A1 was significantly associated with worse PFS $(P=0.009)$. Further stratification analysis revealed that this combined BCSC phenotype was an independent prognostic factor for PFS in some subgroups. In conclusion, we demonstrated the existence of ITSH in TNBC and found that the ITSH as well as a single BCSC marker was not significantly associated with survival, whereas combing the analysis of BCSC markers could improve prognostic value. Our findings may lead to an improvement of prognostic indicators in TNBC.
\end{abstract}

Key words: breast cancer stem cell, intratumor stemness heterogeneity, prognosis, triple-negative breast cancer

\section{Introduction}

Breast cancer is a heterogeneous disease which consists of distinct subtypes, with diverse clinical presentation, response to therapy and prognosis. Triple-negative breast cancer (TNBC) is one of the subtypes characterized by negative for estrogen receptor (ER), progesterone receptor (PR), and human epidermal growth factor receptor-2 (HER2). Currently, no effective endocrine therapy or specific targeted therapy is readily available for TNBC, and furthermore, development of resistance to cytotoxic chemotherapy and early relapse is more common in TNBC than other subtypes [1, 2]. Hence, there is an urgent need for identification of novel biomarkers for TNBC targeted therapy and prognosis.
It has been recently suggested that breast cancer stem cells (BCSCs), which possess the capacity for self-renewal and differentiation, are responsible for tumor initiation, proliferation and progression [3]. The fundamental properties of BCSCs are under the control of complex molecular mechanism in a highly regulated fashion, with influence from the surrounding microenvironment $[4,5]$. The interaction and cross-talk between cancer cells and their microenvironment can impact tumor diversity [6], and the CSC state plasticity can be mediated by the tumor microenvironment through cytokine and chemokine signaling [7]. A number of markers such as CD44, CD24, aldehyde dehydrogenase 1 (ALDH1), 
and CD133 have been proposed for the identification of BCSCs [8-10]. The first tumorigenic BCSCs were distinguished by cells strongly expressed the adhesion molecule CD44 together with no or very low levels of CD24, referred to as $\mathrm{CD} 44^{+} / \mathrm{CD} 24-$ phenotype [8]. It has been reported that $\mathrm{CD} 44^{+} / \mathrm{CD} 24^{-}$ phenotype is associated with poor prognosis in breast cancer [11-13], but some other studies do not confirm this data [14, 15]. The contradicting results of CD44 $/$ CD24- phenotype in prognosis call for additional BCSC markers. ALDH1, a detoxifying enzyme involved in catalyzing the oxidation of intracellular aldehydes, has been suggested as another putative BCSC marker [9]. ALDH1+ phenotype was found to be associated with biological aggressiveness and poor outcomes of breast cancer [9, 16, 17]. Notably, several reports indicated that CD44+/CD24- and ALDH1+ breast cancer cells were associated with TNBC subtype [18-21]. CD133, a transmembrane glycoprotein, is a more recent BCSC marker identified to be correlated with prognosis in breast cancer. Studies in vitro have proven that CD133 is suitable for enriching BCSCs in TNBC $[22,23]$. For CD133+ phenotype, some studies documented poor prognosis in TNBC [24], while others did not [25]. As the expression of these BCSC markers varied among different breast cancer subtypes, it appeared that each BCSC marker could have distinct clinical significance in different subgroups of breast cancers. Considering these controversial issues, continuous evaluations and studies are needed to determine the prognostic value of BCSC phenotypes in breast cancer. Moreover, expression of these BCSC markers seemingly stochastically altered in space [26]. The intratumor genetic heterogeneity mapped to their regional distributions reflects the evidence of tumor evolution, and the prognostic gene-expression signatures assessed from a single region of a heterogeneity tumor may not correctly predict outcomes [27]. Thus whether the intratumor stemness heterogeneity (ITSH) indicates the degrees of malignancy and has prognostic value need to be verified.

In this study, we determined the $\mathrm{CD} 44^{+} / \mathrm{CD}^{-} 4^{-}$, ALDH1A1+ and CD133+ phenotypes as well as the ITSH in TNBC tissue samples, and evaluated their potential prognostic significance. We also combined the analysis of BCSC markers to improve their prognostication in survival.

\section{Materials and methods}

\section{Patients and tissue samples}

Tissue samples from mastectomy and lumpectomy specimens of 88 invasive ductal carcinoma cases were included in this study. These patients were diagnosed between 2005 and 2014. Inclusion criteria were female sex, original histological diagnosis of invasive breast carcinoma, negativity for ER, PR and HER2, without distant metastasis at the time of diagnosis, without neoadjuvant chemotherapy, and availability of clinical data and paraffin blocks. Lack of expression for ER, PR, and HER2 was confirmed by a new immunohistochemical study. Three different anatomic regions from the most representative area of each tumor were obtained to make into tissue microarrays (TAMs) for subsequent processing.

\section{Immunohistochemistry (IHC)}

Expression of ER, PR, HER2, Ki-67, ALDH1A1, and CD133 was analyzed using IHC on serial $4 \mu \mathrm{m}$ tissue sections from TAMs. Paraffin slides were deparaffinized in xylene three times for $10 \mathrm{~min}$ and rehydrated in a graded ethanol series before incubation with $3 \%$ hydrogen peroxide for $10 \mathrm{~min}$ at room temperature to block endogenous peroxidase activity. Antigen retrieval was induced by $10 \mathrm{mM}$ citrate buffer $(\mathrm{pH} 6.0)$ at $98^{\circ} \mathrm{C}$ for $15 \mathrm{~min}$ in a microwave oven. Sections were incubated with $150 \mu \mathrm{L}$ of primary antibody optimally diluted in antibody diluent at $4^{\circ} \mathrm{C}$ overnight in a humidified chamber. The antibodies and dilutions used were: CONFIRM anti-ER (Roche, 790-4325), CONFIRM anti-PR (Roche, 790-4296), VENTANA anti-HER2 (Roche, 790-4493), anti-Ki-67 (Abcam, ab16667) at 1:100 dilution, anti-ALDH1A1 (Abcam, ab52492) at 1:200 dilution, and anti-CD133 (Abnova, MAB10525) at 1:400 dilution. Antibody staining was visualized with 3,3'-diaminobenzidine (DAB) and counterstained with hematoxylin.

\section{Double-staining immunohistochemistry}

Double-staining immunostaining with antibodies for detection of CD44 and CD24 was performed by Double Staining Polymer Detection Kit (ZSGB-BIO, China, DS-0002) according to the manufacturer's instructions. Deparaffinization, rehydration and antigen retrieval were achieved by protocols as mentioned before. Sections were incubated with $150 \mu \mathrm{L}$ mixed primary monoclonal antibodies for CD44 (Abcam, ab51037) at 1:100 dilution and CD24 (Abcam, ab31622) at 1:50 dilution at $37^{\circ} \mathrm{C}$ for $2 \mathrm{~h}$. After washing, mixed biotin-labeled secondary antibody (anti-rabbit and mouse) was applied for $20 \mathrm{~min}$ at room temperature. Color was developed by incubation with permanent-red and DAB respectively.

\section{Immunohistochemical evaluation}

The staining evaluation was performed twice by a pathologist in a blinded fashion. Cells with red color 
staining without much interference from brown color were identified as $\mathrm{CD} 44^{+} / \mathrm{CD} 24^{-}$. ALDH1A1 and CD133 were presented as brown color. The proportion of $\mathrm{CD} 44^{+} / \mathrm{CD} 24-$, ALDH1A1+, and CD133+ tumor cells was estimated from the tumor areas. The scoring protocol was as follows: for CD44 ${ }^{+} / \mathrm{CD} 24-$, staining in $>10 \%$ of tumor cells was considered as positive [14, 15, 28], whereas for ALDH1A1 and CD133, case with $\geq 10 \%$ staining was grouped as positive [29-31]. A case showing positive expression in two or more regions of the three different anatomic regions of each tumor was considered as positive. The ITSH was evaluated regionally according to the expression levels among the three different representative areas made into TAMs. If the three regions from one person showed different expression patterns, it was considered to display ITSH (i.e., one region was positive whereas the other two regions were negative, or one region was negative whereas the other two regions were positive), otherwise considered as non-ITSH (i.e., all three regions were positive, or all three regions were negative). The combined BCSC phenotypes were determined if any marker from the two or three BCSC markers we want to combine was positive (i.e., $\mathrm{CD}_{4} 4^{+} / \mathrm{CD} 24^{-}$and/or ALDH1A1+, $\mathrm{CD}_{4} 4^{+} / \mathrm{CD} 24^{-}$ and/or CD133+, ALDH1A1+ and/or CD133+, and $\mathrm{CD}_{4}{ }^{+} / \mathrm{CD} 24^{-}$and/or ALDH1A1+ and/or CD133+).

\section{Statistical analysis}

Association between the presence of $\mathrm{CD}_{4}{ }^{+} / \mathrm{CD}_{24}-$, ALDH1A1+, and CD133+ phenotypes and clinical variables was assessed by Fisher's exact test. Correlation between different BCSC markers was assessed using Spearman's rank correlation. A log-rank test was used to compare survival between groups in Kaplan-Meier survival plots. Both univariate and multivariate Cox proportional-hazards model were used to investigate association with survival, providing a hazard ratio (HR) and 95\% confidence interval (CI) for each variable. Statistical analysis was carried out using SPSS (v22). All tests were two-sided and $P<0.05$ was considered statistically significant.

\section{Results}

\section{Patient characteristics}

A total of 88 invasive TNBC patients were included in this cohort. Apart from three patients lost to follow-up, the median follow-up period was 72 months (range 10-134 months). The median age of patients was 49 years (range 30-76 years). Of the total, $47(53.4 \%)$ patients were under 50 years of age, and 41 $(46.6 \%)$ patients were over 49 years of age. The median tumor size was $3.0 \mathrm{~cm}$ (range 1.0-6.1 cm).
Eighty $(90.9 \%)$ cases were histological grade 1 or 2, only $8(9.1 \%)$ cases were histological grade 3 . Out of the 88 patients, $82(93.2 \%)$ patients received adjuvant chemotherapy, and $6(6.8 \%)$ patients did not receive any adjuvant treatment. The details of patient clinicopathologic features are summarized in Table 1.

Table 1. Baseline characteristics of the patients

\begin{tabular}{lll}
\hline Variables & Number & $\%$ \\
\hline Age (years) & & \\
$\quad<50$ & 47 & 53.4 \\
$\quad \geq 50$ & 41 & 46.6 \\
Tumor size (cm) & & \\
$\quad \leq 2.0$ & 29 & 33.0 \\
$\quad>2.0$ & 59 & 67.0 \\
Lymph node & & \\
$\quad$ Negative & 51 & 58.0 \\
$\quad$ Positive & 37 & 42.0 \\
Grade & & \\
$\quad 1-2$ & 80 & 90.9 \\
$\quad 3$ & 8 & 9.1 \\
Stage & & \\
$\quad$ I-II & 74 & 84.1 \\
$\quad$ III & 14 & 15.9 \\
Ki-67 (\%) & & \\
$\quad<30$ & 53 & 60.2 \\
$\quad \geq 30$ & 35 & 39.8 \\
Adjuvant chemotherapy & & \\
$\quad$ No & 6 & 6.8 \\
$\quad$ Yes & 82 & 93.2 \\
\hline
\end{tabular}

\section{Expression of BCSC markers}

CD44+/CD24-, ALDH1A1+, and CD133+ tumor cells were detected in human TNBC tissues by IHC staining. Representative staining results are shown in Fig. 1. CD44 showed membranous staining identified by red, and CD24 showed membranous and/or cytoplasmic staining identified by brown. CD133 was found mainly on the membrane and cytoplasm of tumor cells, whereas ALDH1A1 was found mainly on the cytoplasm of tumor cells. Among the 88 primary tumors, $\mathrm{CD}_{4} 4^{+} / \mathrm{CD} 24-$ phenotype was found in 22 $(25.0 \%)$ patients, while $22(25.0 \%)$ and $26(29.5 \%)$ patients expressed ALDH1A1 and CD133, respectively. Patients displayed distinct staining patterns in terms of $\mathrm{CD}_{4} 4^{+} / \mathrm{CD} 24-$, ALDH1A1, and CD133. Eight cases were positive for both CD44 ${ }^{+} / \mathrm{CD} 24-$ and ALDH1A1, 10 case were positive for both $\mathrm{CD}_{4} 4^{+} / \mathrm{CD} 24-$ and $\mathrm{CD} 133,9$ case were positive for both ALDH1A1 and CD133, while only 4 cases showed positivity of all the three BCSC markers.

\section{Intratumor heterogeneity of BCSC markers}

Different anatomic regions from one person could show different expression patterns of BCSC markers, and we defined this heterogeneity as ITSH in our study (Fig. 2). The ITSH of BCSC markers was unable to evaluate in one patient because two tissue samples were lost during the IHC process. Notably, 
only a few patients displayed ITSH within the tumor. In most cases, the three anatomic regions from one specimen exhibited the identical results in the expression of the BCSC markers. The distribution of clinicopathologic features, BCSC markers and ITSH is illustrated as a heatmap in Fig. 3.

\section{Association between BCSC phenotypes and clinicopathologic parameters}

The correlation between the BCSC phenotypes and the clinicopathologic features in TNBC is summarized in Table 2. In our study, no significant correlation was found between BCSC phenotypes and age, tumor size, lymph node metastasis, grade, stage, and Ki-67. Additionally, there was a trend of positive correlation between these BCSC markers, but the correlation did not reach a statistical significance
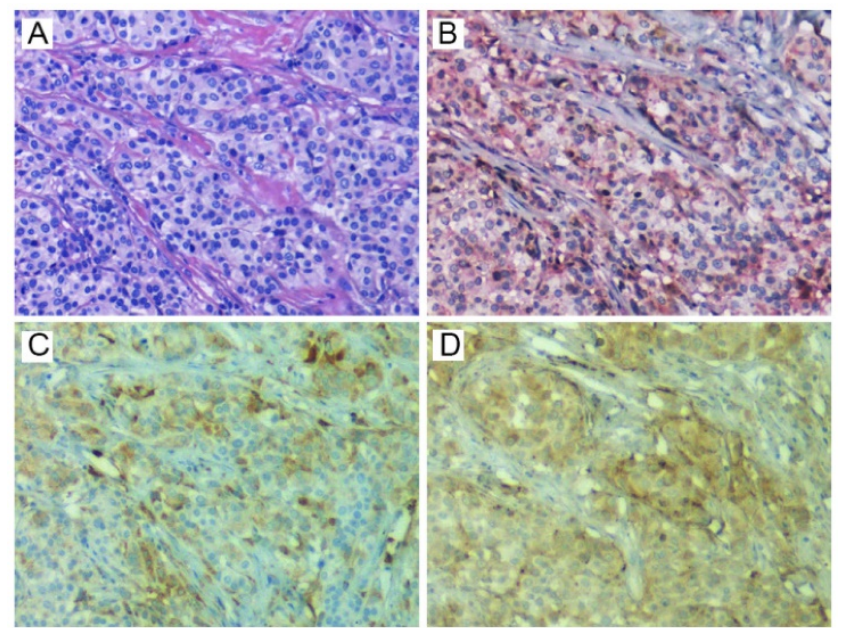

Figure 1. Representative staining of HE (A), CD24 (brown) and CD44 (red) (B), ALDHIAl (C), and CD133 (D). HE, hematoxylin-eosin; ALDHIA1, aldehyde dehydrogenase 1 family member $\mathrm{Al}$. (Table 3).

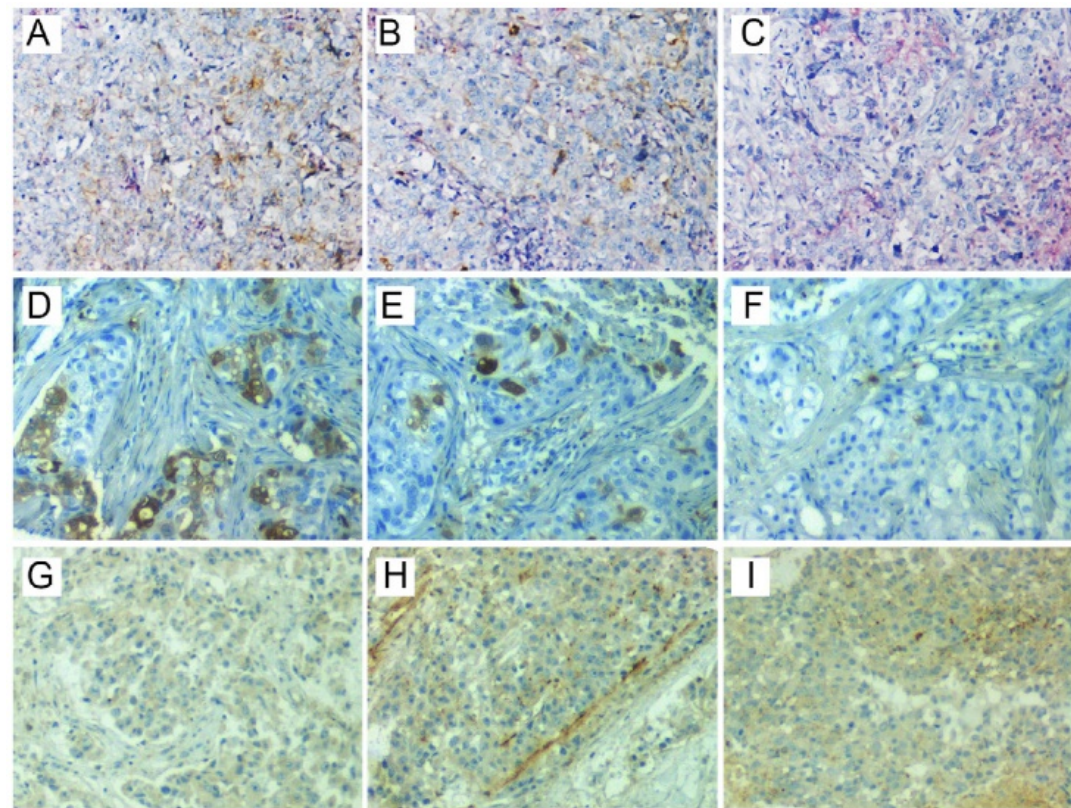

Figure 2. Representative staining of ITSH in three patients. CD44 $/ C D 24$ - was positive in one region (C), but positive in the other two regions (A and B) of the first patient $(A-C)$. ALDHIAl was positive in two regions (D and $E)$, but negative in the other region $(F)$ of the second patient (D-F). CDI33 was positive in two regions $(H$ and $I)$, but negative in the other region $(\mathrm{G})$ of the third patient (G-I). ITSH, intratumor stemness heterogeneity; ALDHIAI, aldehyde dehydrogenase 1 family member $\mathrm{Al}$.

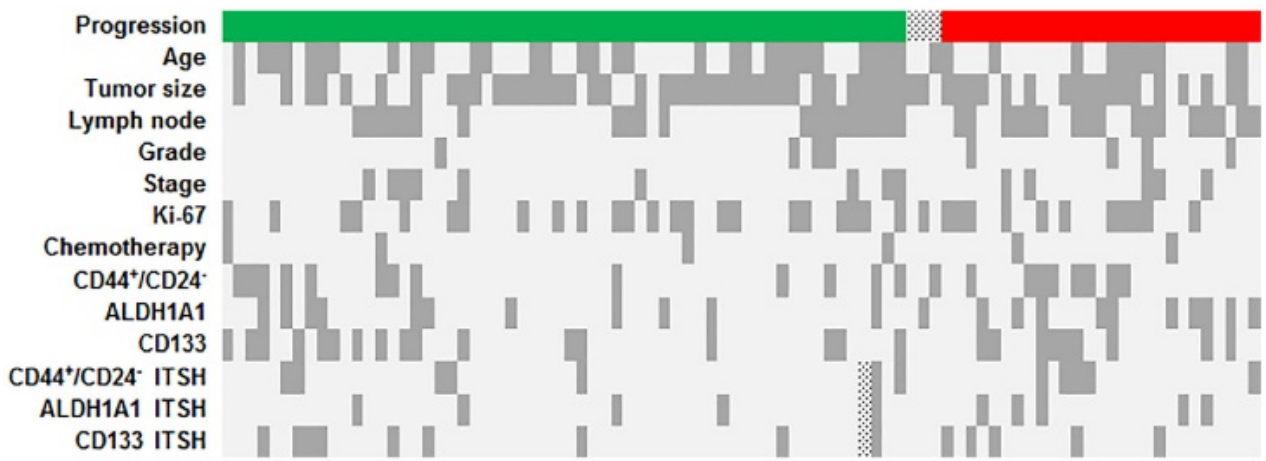

Figure 3. Heatmap showing distribution of progression, clinicopathologic features, BCSC markers, and ITSH in the patients. In the first row, the green cells represented the patients were free of local recurrence and distant metastasis during the follow-up, and the red cells represented that progression occurred during the follow-up, while the dotted cells represented the patients lost to follow-up. In the other rows, the dark gray cells represented that age $\geq 50$ years old, tumor size $>2 \mathrm{~cm}$, positive lymph node, histological grade 3, TNM stage III, Ki-67 $\geq 30 \%$, without receiving adjuvant chemotherapy, positivity of CD44+/CD24-, ALDHIA1 and CDI33 expression, and with ITSH for CD44+/CD24-, ALDHIAI and CDI33 expression. The dotted cells represented the evaluable ITSH status. BCSC, breast cancer stem cell; ALDHIA1, aldehyde dehydrogenase 1 family member $\mathrm{Al}$; ITSH, intratumor stemness heterogeneity. 
Table 2. The association between clinicopathologic variables and BCSC phenotypes

\begin{tabular}{|c|c|c|c|c|c|c|c|c|c|}
\hline \multirow[t]{2}{*}{ Variables } & \multicolumn{2}{|c|}{$\mathrm{CD}_{4} 4^{+} / \mathrm{CD} 24^{-}$} & \multirow[t]{2}{*}{$P$ value } & \multicolumn{2}{|c|}{ ALDH1A1 } & \multirow[t]{2}{*}{$P$ value } & \multicolumn{2}{|c|}{ CD133 } & \multirow[t]{2}{*}{$P$ value } \\
\hline & - & + & & - & + & & - & + & \\
\hline Age (years) & & & 0.085 & & & 1.000 & & & 0.357 \\
\hline$<50$ & 39 & 8 & & 35 & 12 & & 31 & 16 & \\
\hline$\geq 50$ & 27 & 14 & & 31 & 10 & & 31 & 10 & \\
\hline Tumor size $(\mathrm{cm})$ & & & 1.000 & & & 0.795 & & & 0.135 \\
\hline$\leq 2.0$ & 22 & 7 & & 21 & 8 & & 17 & 12 & \\
\hline$>2.0$ & 44 & 15 & & 45 & 14 & & 45 & 14 & \\
\hline Lymph node & & & 0.215 & & & 0.805 & & & 1.000 \\
\hline Negative & 41 & 10 & & 39 & 12 & & 36 & 15 & \\
\hline Positive & 25 & 12 & & 27 & 10 & & 26 & 11 & \\
\hline Grade & & & 0.407 & & & 1.000 & & & 0.689 \\
\hline $1-2$ & 61 & 19 & & 60 & 20 & & 57 & 23 & \\
\hline 3 & 5 & 3 & & 6 & 2 & & 5 & 3 & \\
\hline Stage & & & 0.743 & & & 0.503 & & & 0.750 \\
\hline I-II & 56 & 18 & & 54 & 20 & & 53 & 21 & \\
\hline III & 10 & 4 & & 12 & 2 & & 9 & 5 & \\
\hline Ki-67 (\%) & & & 0.212 & & & 0.079 & & & 1.000 \\
\hline$<30$ & 37 & 16 & & 36 & 17 & & 37 & 16 & \\
\hline$\geq 30$ & 29 & 6 & & 30 & 5 & & 25 & 10 & \\
\hline
\end{tabular}

BCSC, breast cancer stem cell; ALDH1A1, aldehyde dehydrogenase 1 family member A1.

Table 3. Contingency table for BCSC markers by Spearman's rank correlation

\begin{tabular}{llll}
\hline$r(P$ value $)$ & CD44 $^{+} /$CD24- & ALDH1A1 & CD133 \\
\hline CD44 $^{+} /$CD24- & & $0.152(0.159)$ & $0.201(0.060)$ \\
ALDH1A1 & $0.152(0.159)$ & & $0.144(0.181)$ \\
CD133 & $0.201(0.060)$ & $0.144(0.181)$ & \\
\hline
\end{tabular}

BCSC, breast cancer stem cell; ALDH1A1, aldehyde dehydrogenase 1 family member A1.

\section{Survival analysis}

Among the study patients, local recurrence or distant metastasis was found in 27 patients during the study period. Kaplan-Meier survival analysis was performed to explore the association between progression-free survival (PFS) and various clinicopathologic features, such as age, tumor size, lymph node metastasis, grade, stage, Ki-67, adjuvant chemotherapy, BCSC phenotypes, and ITSH. The lymph node-positive groups showed a poorer PFS $(P$ $=0.004)$, and further studies by univariate analysis (HR 3.12, 95\% CI 1.39 to 7.03, $P=0.006$ ) and multivariate analysis (HR 2.98, 95\% CI 1.31 to $6.75, P=$ 0.009 ) had the same results. However, we did not recognize any statistically significant association between the other parameters and PFS. Although patients with ALDH1A1+ phenotype had a trend of increased PFS in multivariate analysis, the difference did not reach a statistical significance (HR 2.19, 95\% CI 0.98 to $4.90, P=0.057$ ) (Table 4 ).

We further grouped patients based on the combined BCSC phenotypes by two or three markers. We defined BCSC phenotype when one or more BCSC markers were positive and non-BCSC phenotype if both or all markers were negative. Interestingly, when we combined CD44 ${ }^{+} / \mathrm{CD} 24^{-}$and ALDH1A1, BCSC phenotype was significantly associated with worse PFS comparing to non-BCSC phenotype (HR 2.76, $95 \%$ CI 1.25 to $6.11, P=0.009)$. However, in other combinations, i.e., $\mathrm{CD} 44^{+} / \mathrm{CD} 24^{-}$and $\mathrm{CD} 133$, ALDH1A1 and CD133, or with all the three markers, no significant association was observed between BCSC phenotypes and PFS (Fig. 4). In the subgroup analysis, the BCSC phenotype combined with CD44 ${ }^{+}$/CD24- and ALDH1A1 was an independent prognostic factor for worse PFS in the patients with younger age (HR 6.09, 95\% CI 1.96 to $18.94, P=0.002$ ), larger tumor size (HR 2.67, 95\% CI 1.01 to $7.06, P=$ 0.048 ), negative lymph node (HR 5.13, 95\% CI 1.27 to 20.67, $P=0.021$ ), earlier histological grade (HR 2.56, 95\% CI 1.09 to $6.02, P=0.031$ ), earlier stage (HR 3.41, $95 \% \mathrm{CI} 1.37$ to $8.50, P=0.008)$, and higher Ki-67 proportion (HR 4.82, 95\% CI 1.38 to $16.81, P=0.014$ ) (Fig. 5).

\section{Discussion}

The cancer stem cell (CSC) hypothesis was coined to describe a rare population of tumorigenic cells that resided at the apex of a hierarchical organization, displaying stem cell properties. The CSC theory has a profound impact on the understanding of breast cancer biology including tumor initiation, metastasis and resistance [3]. Recent works have identified distinct types of BCSCs by various cell surface markers [32]. However, the clinical relevance of these BCSCs remains controversial and there are limited studies exploring the BCSC markers in TNBC. Also, it is not clear whether the BCSC populations identified by different markers represent the same population or 
heterogeneous populations, and whether the spatial heterogeneity within the tumor has an impact on survival. In the present study, we determined the $\mathrm{CD}_{4}{ }^{+} / \mathrm{CD}_{24}-$, ALDH1A1+ + and CD133+ BCSC phenotypes in a cohort of primary TNBCs by IHC. We found that different BCSC markers showed distinct patterns of expression, and a few patients displayed spatial heterogeneity of BCSC markers within the tumor. However, the ITSH was not correlated with survival. Notably, although no significant association between BCSC phenotypes and PFS was found when analyzing BCSC markers separately, it became an independent predictor of PFS when using a composite BCSC phenotype with $\mathrm{CD} 44^{+} / \mathrm{CD} 24{ }^{-}$and ALDH1A1.

Table 4. Univariate and multivariate Cox regression analysis of factors associated with PFS

\begin{tabular}{|c|c|c|c|c|c|}
\hline \multirow[t]{2}{*}{ Variables } & \multirow{2}{*}{$\begin{array}{l}\text { Log-rank } P \\
\text { value }\end{array}$} & \multicolumn{2}{|l|}{ Univariate } & \multicolumn{2}{|l|}{ Multivariate } \\
\hline & & $\mathrm{HR}(95 \% \mathrm{CI})$ & $P$ value & HR $(95 \%$ CI) & $P$ value \\
\hline Age (years) & 0.410 & & & & \\
\hline$<50$ & & 1.00 & & 1.00 & \\
\hline$\geq 50$ & & $0.72(0.33-1.59)$ & 0.414 & $0.69(0.31-1.54)$ & 0.368 \\
\hline Tumor size $(\mathrm{cm})$ & 0.844 & & & & \\
\hline$\leq 2.0$ & & 1.00 & & 1.00 & \\
\hline$>2.0$ & & $0.92(0.41-2.06)$ & 0.845 & $0.97(0.43-2.21)$ & 0.950 \\
\hline Lymph node & 0.004 & & & & \\
\hline Negative & & 1.00 & & 1.00 & \\
\hline Positive & & $3.12(1.39-7.03)$ & 0.006 & $2.98(1.31-6.75)$ & 0.009 \\
\hline Grade & 0.150 & & & & \\
\hline $1-2$ & & 1.00 & & 1.00 & \\
\hline 3 & & $2.14(0.74-6.23)$ & 0.161 & $2.45(0.82-7.31)$ & 0.108 \\
\hline Stage & 0.427 & & & & \\
\hline I-II & & 1.00 & & 1.00 & \\
\hline III & & $1.48(0.56-3.94)$ & 0.432 & $1.61(0.59-4.36)$ & 0.350 \\
\hline Ki-67 (\%) & 0.894 & & & & \\
\hline$<30$ & & 1.00 & & 1.00 & \\
\hline$\geq 30$ & & $0.95(0.43-2.09)$ & 0.894 & $0.93(0.42-2.07)$ & 0.859 \\
\hline Adjuvant chemotherapy & 0.687 & & & & \\
\hline No & & 1.00 & & 1.00 & \\
\hline Yes & & $0.74(0.18-3.16)$ & 0.689 & $0.74(0.17-3.20)$ & 0.691 \\
\hline \multicolumn{6}{|l|}{ BCSC phenotype } \\
\hline & 0.248 & & & & \\
\hline non-CD44+/CD24- & & 1.00 & & 1.00 & \\
\hline \multirow[t]{2}{*}{$\mathrm{CD}_{4} 4^{+} / \mathrm{CD} 24^{-}$} & & $1.63(0.71-3.75)$ & 0.255 & $1.74(0.73-4.13)$ & 0.211 \\
\hline & 0.088 & & & & \\
\hline ALDH1A1- & & 1.00 & & 1.00 & \\
\hline \multirow[t]{2}{*}{ ALDH1A1+ } & & $1.96(0.89-4.33)$ & 0.095 & $2.19(0.98-4.90)$ & 0.057 \\
\hline & 0.597 & & & & \\
\hline CD133- & & 1.00 & & 1.00 & \\
\hline CD133+ & & $1.24(0.56-2.77)$ & 0.599 & $1.10(0.49-2.50)$ & 0.813 \\
\hline $\mathrm{CD}_{4} 4^{+} / \mathrm{CD} 24-$ and/or ADLH1A1+ & 0.009 & & & & \\
\hline No & & 1.00 & & 1.00 & \\
\hline Yes & & $2.76(1.25-6.11)$ & 0.012 & $2.81(1.26-6.24)$ & 0.011 \\
\hline $\mathrm{CD}_{4} 4^{+} / \mathrm{CD} 24-$ and/or CD133+ & 0.348 & & & & \\
\hline No & & 1.00 & & 1.00 & \\
\hline Yes & & $1.44(0.67-3.08)$ & 0.352 & $1.41(0.66-3.03)$ & 0.381 \\
\hline ALDH1A1+ and/or CD133+ & 0.349 & & & & \\
\hline No & & 1.00 & & 1.00 & \\
\hline Yes & & $1.43(0.67-3.06)$ & 0.354 & $1.43(0.66-3.09)$ & 0.361 \\
\hline \multicolumn{6}{|c|}{$\mathrm{CD}_{4} 4^{+} / \mathrm{CD} 24-$ and/or ALDH1A1+ and/or CD133+ 0.057} \\
\hline No & & 1.00 & & 1.00 & \\
\hline Yes & & $2.15(0.96-4.84)$ & 0.063 & $2.18(0.97-4.90)$ & 0.061 \\
\hline $\mathrm{CD} 44^{+} / \mathrm{CD} 24-\mathrm{ITSH}$ & 0.620 & & & & \\
\hline No & & 1.00 & & 1.00 & \\
\hline Yes & & $1.28(0.48-3.38)$ & 0.622 & $1.27(0.48-3.38)$ & 0.634 \\
\hline ALDH1A1 ITSH & 0.238 & & & & \\
\hline No & & 1.00 & & 1.00 & \\
\hline Yes & & $1.78(0.67-4.75)$ & 0.247 & $1.88(0.68-5.19)$ & 0.225 \\
\hline CD133 ITSH & 0.546 & & & & \\
\hline No & & 1.00 & & 1.000 & \\
\hline Yes & & $1.35(0.51-3.58)$ & 0.549 & $1.91(0.65-5.56)$ & 0.237 \\
\hline
\end{tabular}

PFS, progression-free survival; BCSC, breast cancer stem cell; ALDH1A1, aldehyde dehydrogenase 1 family member A1; ITSH, intratumor stemness heterogeneity; HR, hazard ratio; CI, confidence interval. 
A

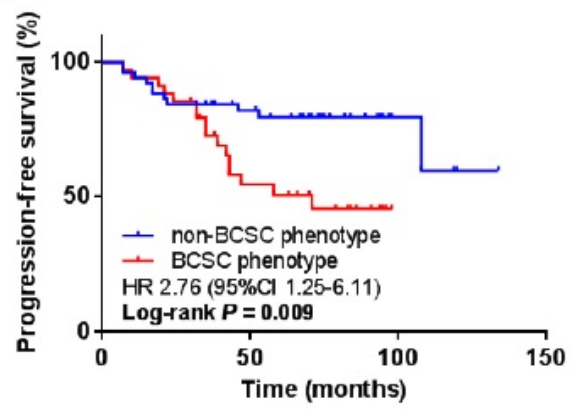

C

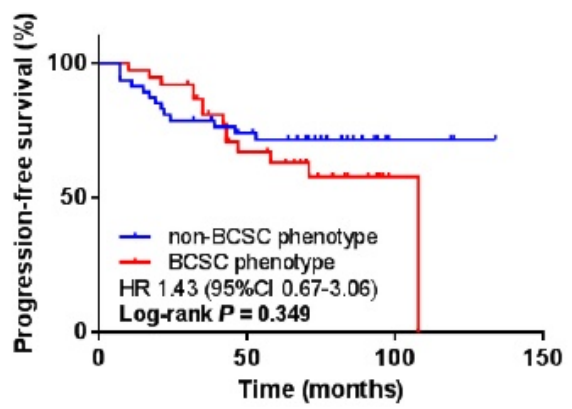

B

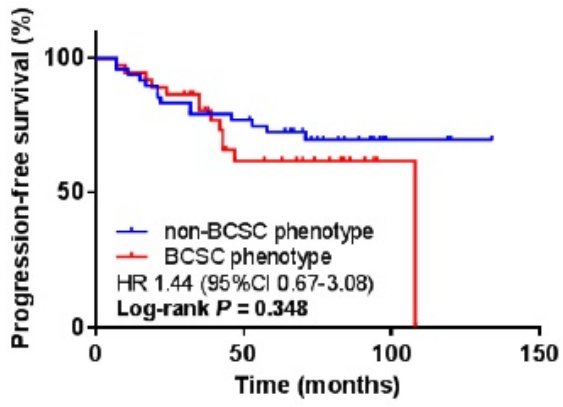

D

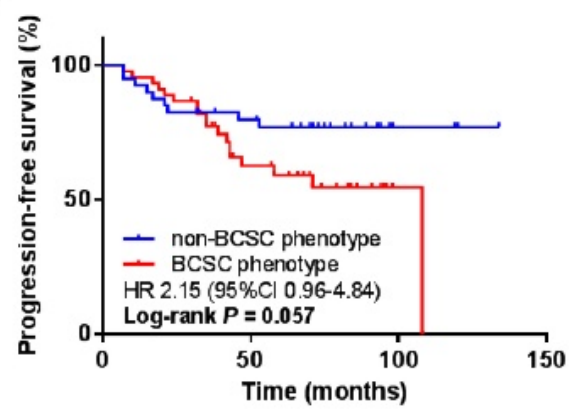

Figure 4. Kaplan-Meier survival analysis of the combined BCSC phenotypes with PFS. The BCSC phenotypes were determined by combing the expression of

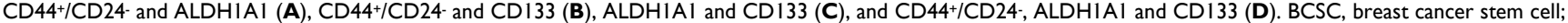
PFS, progression-free survival; ALDH1A1, aldehyde dehydrogenase 1 family member A1.

$\mathrm{HR}(95 \% \mathrm{Cl})$

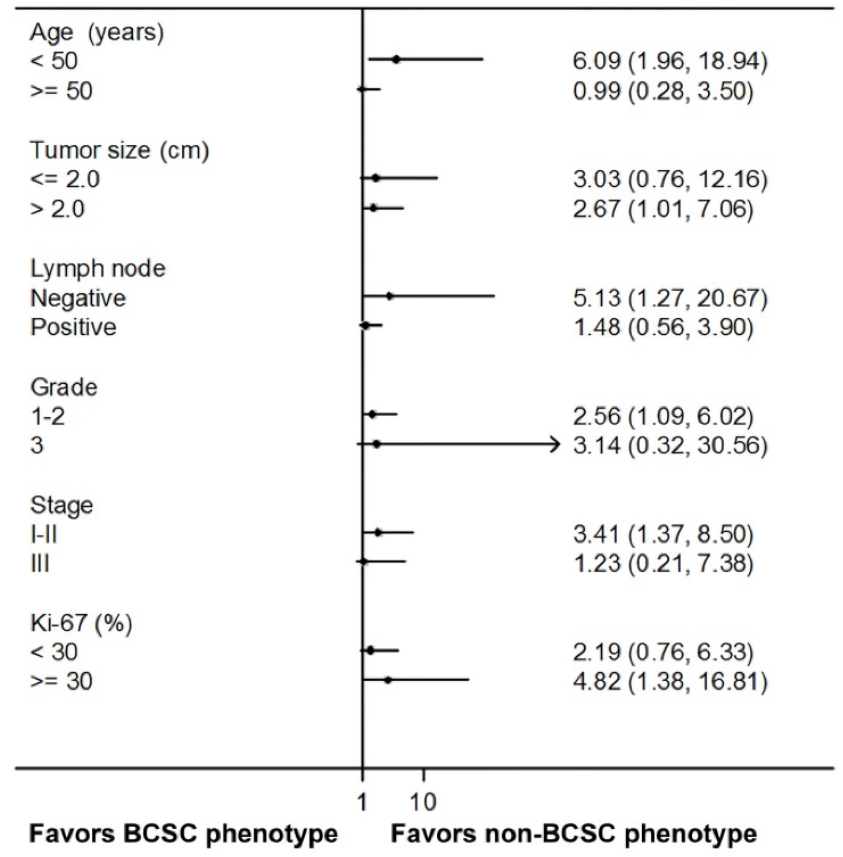

Figure 5. Subgroup analysis of PFS by BCSC phenotypes combined with CD44 ${ }^{+}$CD 24- and ALDHIAI. PFS, progression-free survival; BCSC, breast cancer stem cell; ALDHIAI, aldehyde dehydrogenase 1 family member Al.

The $\mathrm{CD}_{4} 4^{+} / \mathrm{CD} 24^{-}$phenotype was the first marker determined to enrich for BCSCs [8]. The high proportion of $\mathrm{CD}_{4} 4^{+} / \mathrm{CD} 24-$ tumor cells in breast cancer was thought to be correlated with negative lymph node [14], distant metastasis [15], and poor prognosis [11-13]. However, we did not find any association with the clinical features including age, tumor size, lymph node status, histological grade, TNM stage and Ki-67. The discordance may ascribe to the different subtypes of breast cancer we studied, as some recent reports have not yet shown any association with the clinicopathologic variables in TNBC $[28,33]$. There are limited data indicating the prognostic role of $\mathrm{CD}_{4} 4^{+} / \mathrm{CD} 24^{-}$phenotype in TNBC. Here, we found that there was no significant difference in survival between patients with and without CD44 ${ }^{+}$CD24- phenotype.

ALDH1 has come to the forefront as an additional BCSC marker since the role of $\mathrm{CD}_{4}{ }^{+} / \mathrm{CD} 24^{-}$remains controversial. Ginestier et al. firstly identified ALDH1 as a specific marker assessed by ADELFLUOR assay as well as IHC for the enrichment of BCSCs [9], and it prompted a series of attempts to characterize the relationship between ALDH1+ phenotype and clinicopathologic features in breast cancer. However, conclusions reported by different investigators seem to be inconsistent [34-36]. Here we did not find any association between ALDH1A1 expression and clinicopathologic features in TNBC. Although ALDH1 had a significant impact on prognosis in the other subtypes, it did not appear to correlate with the survival of TNBC patients [37-39]. Here we showed a borderline significant trend of ALDH1A1+ phenotype for PFS. The heterogeneous results may have some possible 
explanations. First, the cutoff value was defined differently $(0 \%, 5 \%, 10 \%, 20 \%$, or other classification) in these studies $[40,41]$, which could have an impact on the positivity of ALDH1. Second, the approaches applied to detect ALDH1+ BCSCs were different [36], and thereby it did not coincide completely with the populations identified by ADELFLUOR assay and IHC. Third, ALDH1 has three main isoforms (ALDH1A1, ALDH1A2, and ALDH1A3), although ALDH1A1 was thought to be the major contributor of ALDH1 activity in breast cancer [42], lack of strict distinction of these isoforms in most studies might contribute to the discrepancy to some extent.

CD133, a glycoprotein known as Prominin 1 (PROM1), has been considered as a CSC marker in various solid tumors [43-46]. It has been reported that CD133 expression correlates significantly with TNBC subtype [30,47]. However, the biologic function of CD133 in TNBC has not yet been well established and its prognostic role still remains debated [24, 25]. We did not find any evidence of CD133 in predicting aggressive properties and poor outcome in TNBC. The diverse conclusions from different studies could be due to the extreme heterogeneity of commercial antibodies and cutoff values, or could also be due to TNBC itself being a heterogeneous tumor class.

We found that the patients displayed the similar positive rates of CD44 ${ }^{+}$/CD24-, ALDH1A1 and CD133 expression. Further analysis demonstrated a weak correlation between BCSC markers, implying that the distinct populations determined by these markers have some overlap but most breast cancer cells do not express these BCSC markers concurrently. In addition, the expression of BCSC markers could alter in space within the tumor, although in only a few patients. These findings revealed the ITSH in breast cancer and indicated that BCSC phenotype determined by a single region from a heterogeneous tumor may not totally represent the real stem status of the tumor. It might be a possible explanation for the controversial role of BCSCs in tumor biology and prognosis. Thus multiregional analysis from one tumor could probably provide more accurate information in identifying BCSC phenotype.

Since the limitation of a single marker in identifying BCSCs, the idea of combining markers has been taken by researchers. They found that the combination of $\mathrm{CD}_{4} 4^{+} / \mathrm{CD} 24^{-}$and ALDEFLUOR activity enabled them to isolate tumor cells able to form tumors with fewer numbers compared to the cells sorted by ALDEFLUOR activity alone; however, they did not investigate the clinical implications of this combination [9] . Actually, recent studies have shown that a combined analysis of BCSC markers can robustly predict outcome, but it works only limited to a subset of breast cancer [48-50]. The current study demonstrated that the combined BCSC phenotype with $\mathrm{CD}_{4} 4^{+} / \mathrm{CD} 24^{-}$and ALDH1A1 could be an independent predictor of PFS in TNBC. Moreover, the clinical relevance was also described in subgroups characterized by clinicopathologic features. The results indicated that the subpopulations determined by $\mathrm{CD} 44^{+} / \mathrm{CD} 24^{-}$and ALDH1A1 could both represent BCSCs. Thus it showed a strengthened prognostic value of the combined BCSC markers compared to the single marker, because when we identified the tumor as a non-BCSC phenotype based on a single marker, it could be positive for another BCSC marker and endowed with self-renewal and differentiation capacity actually.

Our study has several potential limitations. The first is the inadequate sample size and the short follow-up duration. A single BCSC marker may actually be associated with clinical outcome, but a larger sample size and a longer observation period are needed to demonstrate such a relationship. Second, we used TMAs to detect BCSC populations, and therefore there was likely to be some sampling error. In fact, we have taken three tumor areas of each specimen to address this problem. Third, we applied IHC instead of flow cytometry to determine the BCSC phenotypes, whereas putative CSCs were originally identified by the latter. Although we assumed that flow cytometry could be translated into IHC based equivalent and expected this change to identify the subpopulation of BCSCs with a high degree of overlap, it is possible that there will be some discordance. Actually, there have been increasing evidences that IHC can be taken as a useful tool to identify BCSCs and shows great potential clinical application value [28, 40,49]. Finally, our analysis did not explore the correlation with overall survival (OS) as it could be influenced by multiple factors during long follow-up durations. Hence, we advise cautious interpretation of the results before further evaluations and studies overcoming the limitations of our current study.

\section{Conclusions}

In summary, we identified diverse BCSC phenotypes in a cohort of TNBCs, and demonstrated the ITSH in a few patients. However, no significant correlation between the ITSH and PFS was found. None of the BCSC markers was associated with PFS when analyzed separately, although there was a trend towards that BCSC phenotypes showed worse survival. We also found that the predictor became more powerful when using a composite BCSC phenotype by $\mathrm{CD}_{4} 4^{+} / \mathrm{CD} 24^{-}$and ALDH1A1. Therefore, combining BCSC markers may have higher 
prognostic value, and thereby can probably promote the discovery of a panel of markers to robustly identify BCSCs in TNBC. However, further validation studies in independent cohorts are required before definitive conclusions can be drawn.

\section{Abbreviations}

TNBC: triple-negative breast cancer; BCSC: breast cancer stem cell; CSC: cancer stem cell; ALDH1: aldehyde dehydrogenase 1; ALDH1A1: aldehyde dehydrogenase 1 family member A1; PFS: progression-free survival; OS: overall survival; ITSH: intratumor stemness heterogeneity; ER: estrogen receptor; PR: progesterone receptor; HER2: human epidermal growth factor receptor-2; TAM: tissue microarray; IHC: immunohistochemistry; HR: hazard ratio; CI: confidence interval

\section{Acknowledgments}

This project is supported by grants from the Natural Science Foundation of China (no. 81470357) and a Foundation for Clinical Medicine Science and Technology Special Project of the Jiangsu Province, China (no. BL2014071) (to X.G).

\section{Competing Interests}

The authors have declared that no competing interest exists.

\section{References}

1. Gluz O, Liedtke C, Gottschalk N, et al. Triple-negative breast cancer--current status and future directions. Ann Oncol. 2009; 20: 1913-27.

2. Ismail-Khan R, Bui MM. A review of triple-negative breast cancer. Cancer Control. 2010; 17: 173-6.

3. Lin $\mathrm{CY}$, Barry-Holson KQ, Allison $\mathrm{KH}$. Breast cancer stem cells: are we ready to go from bench to bedside? Histopathology. 2016; 68: 119-37.

4. Reya T, Morrison SJ, Clarke MF, et al. Stem cells, cancer, and cancer stem cells. Nature. 2001; 414: 105-11.

5. Plaks V, Kong N, Werb Z. The cancer stem cell niche: how essential is the niche in regulating stemness of tumor cells? Cell Stem Cell. 2015; 16: 225-38.

6. Koren S, Bentires-Alj M. Breast Tumor Heterogeneity: Source of Fitness, Hurdle for Therapy. Mol Cell. 2015; 60: 537-46.

7. Brooks MD, Burness ML, Wicha MS. Therapeutic Implications of Cellular Heterogeneity and Plasticity in Breast Cancer. Cell Stem Cell. 2015; 17: 260-71.

8. Al-Hajj M, Wicha MS, Benito-Hernandez A, et al. Prospective identification of tumorigenic breast cancer cells. Proc Natl Acad Sci U S A. 2003; 100: 3983-8.

9. Ginestier C, Hur MH, Charafe-Jauffret E, et al. ALDH1 is a marker of normal and malignant human mammary stem cells and a predictor of poor clinical outcome. Cell Stem Cell. 2007; 1: 555-67.

10. Wright $\mathrm{MH}$, Calcagno $\mathrm{AM}$, Salcido $\mathrm{CD}$, et al. Brca1 breast tumors contain distinct CD44+/CD24- and CD133+ cells with cancer stem cell characteristics. Breast Cancer Res. 2008; 10: R10.

11. Ricardo S, Vieira AF, Gerhard R, et al. Breast cancer stem cell markers CD44, CD24 and ALDH1: expression distribution within intrinsic molecular subtype. J Clin Pathol. 2011; 64: 937-46.

12. Honeth G, Bendahl PO, Ringner M, et al. The CD44+/CD24- phenotype is enriched in basal-like breast tumors. Breast Cancer Res. 2008; 10: R53.

13. Buess M, Rajski M, Vogel-Durrer BM, et al. Tumor-endothelial interaction links the CD44(+)/CD24(-) phenotype with poor prognosis in early-stage breast cancer. Neoplasia. 2009; 11: 987-1002

14. Mylona E, Giannopoulou I, Fasomytakis E, et al. The clinicopathologic and prognostic significance of CD44+/CD24(-/low) and CD44-/CD24+ tumor cells in invasive breast carcinomas. Hum Pathol. 2008; 39: 1096-102.

15. Abraham BK, Fritz P, McClellan M, et al. Prevalence of CD44+/CD24-/low cells in breast cancer may not be associated with clinical outcome but may favor distant metastasis. Clin Cancer Res. 2005; 11: 1154-9.

16. Morimoto K, Kim SJ, Tanei T, et al. Stem cell marker aldehyde dehydrogenase 1-positive breast cancers are characterized by negative estrogen receptor, positive human epidermal growth factor receptor type 2, and high Ki67 expression. Cancer Sci. 2009; 100: 1062-8.

17. Tanei T, Morimoto K, Shimazu K, et al. Association of breast cancer stem cells identified by aldehyde dehydrogenase 1 expression with resistance to sequential Paclitaxel and epirubicin-based chemotherapy for breast cancers. Clin Cancer Res. 2009; 15: 4234-41.

18. Perrone G, Gaeta LM, Zagami M, et al. In situ identification of CD44+/CD24cancer cells in primary human breast carcinomas. PLoS One. 2012; 7: e43110.

19. Sheridan C, Kishimoto H, Fuchs RK, et al. CD44+/CD24- breast cancer cells exhibit enhanced invasive properties: an early step necessary for metastasis. Breast Cancer Res. 2006; 8: R59.

20. Sun H, Jia J, Wang X, et al. CD44+/CD24- breast cancer cells isolated from MCF-7 cultures exhibit enhanced angiogenic properties. Clin Transl Oncol. 2013; 15: 46-54.

21. Guler G, Balci S, Costinean S, et al. Stem cell-related markers in primary breast cancers and associated metastatic lesions. Mod Pathol. 2012; 25: 949-55.

22. Liu TJ, Sun BC, Zhao XL, et al. CD133+ cells with cancer stem cell characteristics associates with vasculogenic mimicry in triple-negative breast cancer. Oncogene. 2013; 32: 544-53.

23. Brugnoli F, Grassilli S, Piazzi M, et al. In triple negative breast tumor cells, PLC-beta2 promotes the conversion of CD133high to CD133low phenotype and reduces the CD133-related invasiveness. Mol Cancer. 2013; 12: 165.

24. Zhao $P$, Lu Y, Jiang $X$, et al. Clinicopathological significance and prognostic value of CD133 expression in triple-negative breast carcinoma. Cancer Sci. 2011; 102: 1107-11.

25. Bock C, Kuhn C, Ditsch N, et al. Strong correlation between N-cadherin and CD133 in breast cancer: role of both markers in metastatic events. J Cancer Res Clin Oncol. 2014; 140: 1873-81.

26. Wei H, Fu P, Yao M, et al. Breast cancer stem cells phenotype and plasma cell-predominant breast cancer independently indicate poor survival. Pathol Res Pract. 2016; 212: 294-301.

27. Gerlinger M, Rowan AJ, Horswell S, et al. Intratumor heterogeneity and branched evolution revealed by multiregion sequencing. N Engl J Med. 2012; 366: 883-92.

28. Ma F, Li H, Wang H, et al. Enriched CD44(+)/CD24(-) population drives the aggressive phenotypes presented in triple-negative breast cancer (TNBC). Cancer Lett. 2014; 353: 153-9.

29. Mansour SF, Atwa MM. Clinicopathological Significance of CD133 and ALDH1 Cancer Stem Cell Marker Expression in Invasive Ductal Breast Carcinoma. Asian Pac J Cancer Prev. 2015; 16: 7491-6.

30. Kim SJ, Kim YS, Jang ED, et al. Prognostic Impact and Clinicopathological Correlation of CD133 and ALDH1 Expression in Invasive Breast Cancer. J Breast Cancer. 2015; 18: 347-55.

31. Aomatsu N, Yashiro M, Kashiwagi S, et al. CD133 is a useful surrogate marker for predicting chemosensitivity to neoadjuvant chemotherapy in breast cancer. PLoS One. 2012; 7: e45865.

32. Badve S, Nakshatri H. Breast-cancer stem cells-beyond semantics. Lancet Oncol. 2012; 13: e43-8.

33. Uchoa Dde M, Graudenz MS, Callegari-Jacques SM, et al. Expression of cancer stem cell markers in basal and penta-negative breast carcinomas--a study of a series of triple-negative tumors. Pathol Res Pract. 2014; 210: 432-9.

34. Zhou Y, Wang Y, Ju X, et al. Clinicopathological significance of ALDH1A1 in lung, colorectal, and breast cancers: a meta-analysis. Biomark Med. 2015; 9: 777-90.

35. Liu JF, Xia P, Hu WQ, et al. Aldehyde dehydrogenase 1 expression correlates with clinicopathologic features of patients with breast cancer: a meta-analysis. Int J Clin Exp Med. 2015; 8: 8425-32.

36. Liu Y, Lv DL, Duan JJ, et al. ALDH1A1 expression correlates with clinicopathologic features and poor prognosis of breast cancer patients: a systematic review and meta-analysis. BMC Cancer. 2014; 14: 444.

37. Kida K, Ishikawa T, Yamada A, et al. Effect of ALDH1 on prognosis and chemoresistance by breast cancer subtype. Breast Cancer Res Treat. 2016; 156: 261-9.

38. Kim YS, Jung MJ, Ryu DW, et al. Clinicopathologic characteristics of breast cancer stem cells identified on the basis of aldehyde dehydrogenase 1 expression. J Breast Cancer. 2014; 17: 121-8.

39. Collina F, Di Bonito M, Li Bergolis V, et al. Prognostic Value of Cancer Stem Cells Markers in Triple-Negative Breast Cancer. Biomed Res Int. 2015; 2015: 158682.

40. Alamgeer M, Ganju V, Kumar B, et al. Changes in aldehyde dehydrogenase-1 expression during neoadjuvant chemotherapy predict outcome in locally advanced breast cancer. Breast Cancer Res. 2014; 16: R44.

41. Gong Y, Wang J, Huo L, et al. Aldehyde dehydrogenase 1 expression in inflammatory breast cancer as measured by immunohistochemical staining. Clin Breast Cancer. 2014; 14: e81-8.

42. Tomita H, Tanaka K, Tanaka T, et al. Aldehyde dehydrogenase $1 \mathrm{~A} 1$ in stem cells and cancer. Oncotarget. 2016; 7: 11018-32.

43. Park E, Park SY, Sun PL, et al. Prognostic significance of stem cell-related marker expression and its correlation with histologic subtypes in lung adenocarcinoma. Oncotarget. 2016.

44. Zhao Y, Peng J, Zhang E, et al. CD133 expression may be useful as a prognostic indicator in colorectal cancer, a tool for optimizing therapy and supportive evidence for the cancer stem cell hypothesis: a meta-analysis. Oncotarget. 2016; 7: 10023-36. 
45. Zhang X, Hua R, Wang X, et al. Identification of stem-like cells and clinical significance of candidate stem cell markers in gastric cancer. Oncotarget. 2016; 7: 9815-31

46. Nomura A, McGinn O, Dudeja V, et al. Minnelide effectively eliminates CD133(+) side population in pancreatic cancer. Mol Cancer. 2015; 14: 200.

47. Currie MJ, Beardsley BE, Harris GC, et al. Immunohistochemical analysis of cancer stem cell markers in invasive breast carcinoma and associated ductal carcinoma in situ: relationships with markers of tumor hypoxia and microvascularity. Hum Pathol. 2013; 44: 402-11.

48. Neumeister V, Agarwal S, Bordeaux J, et al. In situ identification of putative cancer stem cells by multiplexing ALDH1, CD44, and cytokeratin identifies breast cancer patients with poor prognosis. Am J Pathol. 2010; 176: 2131-8.

49. Ali HR, Dawson SJ, Blows FM, et al. Cancer stem cell markers in breast cancer: pathological, clinical and prognostic significance. Breast Cancer Res. 2011; 13: R118.

50. Tsang JY, Huang YH, Luo MH, et al. Cancer stem cell markers are associated with adverse biomarker profiles and molecular subtypes of breast cancer. Breast Cancer Res Treat. 2012; 136: 407-17. 